\title{
Next Directions in Experimental Data for Seismic Hazard Mitigation
}

\begin{abstract}
Data are one of the main assets of earthquake engineering. Laboratory experiments can be extremely expensive and time consuming to replicate and, therefore, long-term preservation of experimental data and sharing the data with users has become one of the disciplinary priorities. There is a growing demand for international partnerships, which creates a need for data sharing, in an attempt to maximise research impact and to tackle experimental set-ups that could not be realised otherwise. However, there is a patent lack of interoperability between the institutions forming the earthquake engineering community, which inhibits efficient collaboration between them. In this paper, we discuss a vision about the directions that experimental data should take in the coming years, focusing on two aspects: enhanced international collaborations and implementation of open data access. We also describe the progress that has been made towards this vision, by establishing an open platform for the integration of earthquake hazard mitigation resources called Celestina. Celestina is supported by Semantic Web technologies, and uses an ontology as its integration data model. A prototype of the platform has been developed and tested between NEES (Purdue University, in the US), the University of Oxford (in UK) and EUCENTRE (in Italy), and a small proof of concept has enabled integrated experimental data from Oxford and EUCENTRE through the NEES cyberenvironment. This demonstration provides an example which has the potential to catalyze a new generation of research progress enabled by international data sharing.
\end{abstract}

\section{INTRODUCTION}

Earthquake engineering is the scientific discipline that studies the nature of earthquakes and their effects on structures, such as buildings or bridges, in order to diminish their damage and with the ultimate goals of saving human life and minimising economic loss. The study of how an earthquake affects different structures and their materials is either conducted by observing the behaviour of structures that have been subjected to a real earthquake, or conducted by means of experiments, where part or a full structure, soil or devices are placed under a seismic stimulus in a laboratory and their behaviour is recorded for subsequent analysis. Experiments provide valuable data for the development of systems to minimise the impact of earthquakes, but their execution can be very costly. Computing advances have enabled new methods of evaluating seismic performance, for example by simulating the behaviour of structures numerically. However, uncertainty over physical parameters and limitations on the understanding of element behaviour can limit the applicability of computer models, requiring the actual physical testing of the structure.

Data collected from real earthquakes and experiments are a highly valued resource in earthquake engineering, since laboratory experiments can be extremely expensive and time consuming to replicate, and the effects of real earthquakes are impossible to record a second time. Besides the need of a formalised way to store experimental data, the expanding demand for international collaborations creates a need for data sharing in an attempt to maximise research efforts and to tackle experiment set-ups that could not be realised otherwise. However, there is a patent lack of interoperability between the institutions forming the earthquake engineering community, which introduces barriers to efficient collaboration between them. Moreover, even 
though there exist some efforts to share experimental data (such as those of NEES ${ }^{1}$ in the US and SERIES $^{2}$ in Europe), this only addresses the consumption of data by humans, since the lack of interoperability prevents automated data consumption.

In this paper, we present a vision about the directions that experimental data should take in the coming years that builds upon the past successes in the earthquake engineering community [e.g.1, 2]. We focus the discussion on two aspects: enhanced international collaborations and implementation of open data policies. We also describe the work we have done towards this vision, by creating a platform for the integration of earthquake hazard mitigation resources.

\section{STATE OF THE ART}

\subsection{Current status of experimental data in earthquake engineering}

The earthquake engineering community has been a leader in the effort to provide open data to accelerate scientific advances needed to build more resilient communities around the world. The most noteworthy examples of experimental data repositories are those of NEES (Network for Earthquake Engineering Simulation) and SERIES (Seismic Engineering Research Infrastructures for European Synergies). The NEES infrastructure [3] has a centralised data repository to store and share experimental data, whose data model is described by [4] and [5]. The quality and completeness of the data are verified and improved by means of a process called data curation, in which the data are monitored and approved for publication [6]. NEES has commitments in terms of digital data preservation, to ensure that data remain accessible and usable [7], and provides open licenses and digital identifiers for both documents and data resulting from experimental activities. The NEES database provided data integration to the earthquake engineering institutions in the USA. The database is supported by a number of tools such as inDEED [8], and PEN [9]. NEES has been superseded by NHERI (Natural Hazards Engineering Research Infrastructure), whose cyberinfrastructure component ${ }^{3}$ is in charge of NEES legacy of data. Changes to the data management model are expected in the next few years.

In Europe, SERIES created the SERIES Virtual Database [10], an infrastructure of distributed data sources that allows access to the SERIES European laboratories' data through a single, centralised user interface, and that enables data integration between the different SERIES institutions. The main reasons for a decentralised approach (instead of centralised) are that European institutions need to be autonomous and have control of their own data; also, a decentralised solution contributes to reduce the technological gap between each laboratory. The distributed nature of the virtual database is intended to be invisible to the end user, whose experience should be similar to accessing a single data repository.

Other databases have been internally created at different institutions around the world, such as at the Joint Research Centre and EUCENTRE in Italy, and E-defense in Japan, but they have not been documented publicly. EPOS $^{4}$ (European Plate Observing System) also provides public access to integrated multidisciplinary Earth science data at a European level, such as data

\footnotetext{
1 https://nees.org

2 http://www.series.upatras.gr

3 https://www.designsafe-ci.org

4 https://www.epos-ip.org/
} 
from volcano observatories, seismic waveform data from ORFEUS ${ }^{5}$ and laboratory experimental data from other European institutions.

A digital archive of video, audio, images and documents related to seismic events across New Zealand has also been established within the CEISMIC center ${ }^{6}$. The number and variety of repositories is evidence of the continued importance of accessing data from experiments to enable research and scientific advances in this field.

\subsection{Data policies}

Globally, data policies on publicly funded research are increasingly moving to an Open Access schema, in which research output is published free to access, redistribute and, in many cases, reuse. Examples of funding bodies requiring or encouraging to publish research outcomes on an Open Access basis are the Research Councils UK [11], Wellcome Trust [12], the US National Science Foundation [13], the US Department of Energy [14] and the US National Institutes of Health [15]. In the UK, HEFCE (Higher Education Funding Council for England, the government agency responsible for university core funding) will require open access publications for the next research assessment process [16]. In the US, the National Science Foundation, which supports about one-quarter of all federally-funded research in the US, has required a data management plan for all research projects since 2013, and the US government recently adopted the Open Government Plan 3.5 [17]. It will not be long before public funding bodies strictly require all their funded publications to be disseminated under an Open Access schema. Open Access is often supported by publishers (e.g. BioMed Central [18], Plos [19], etc.) and repositories (e.g. PubMed Central [20], Europe PMC [21], repositories indexed by OpenAire [22], etc.).

The next step after the publishing industry adopts Open Access is to embrace Open Data. Sources of public data are also moving in the Open Data direction, and good examples are the governments of the USA [23], Europe [24] and Asia [25] amongst others. In Earthquake Engineering, the NEES repository has encouraged the use of Open Data, and nearly all of their experimental outcomes have been published under such schema [26]. Also, other seismic related repositories such as the ones at EPOS and ORFEUS are encouraging the adoption of open data $[27,28]$.

Open Data creates new opportunities for data sharing and reuse, but at the same time imposes additional requirements on infrastructures to enable such interoperability. Data management and publishing systems need to shift their focus accordingly: new tasks of data exchange and information integration are gaining prominence, while traditional concerns such as user rights management and access control are less critical. New types of Open Data management systems should therefore be tailored towards this new form of publishing.

\subsection{Interoperability efforts in other sciences}

Science Gateways have been developed within several scientific domains in an effort to provide essential, often domain-specific, cyberinfrastructure services to researchers to accelerate research and knowledge generation [29]. Some other sciences have also attempted to create a global interoperability environment. For example, there has been an effort in bioinformatics to integrate different data sources that already expose their data in RDF, a Semantic Web technology [30].

\footnotetext{
5 http://www.orfeus-eu.org/

$6 \quad$ http://www.ceismic.org.nz
} 
This effort primarily focused on the integration of three existing datasets by using SPARQL, another Semantic Web technology that is briefly described later, without alteration of the existing data. By using SPARQL, an integrated layer is created and the three repositories can be queried in a unified, uniform manner. One of the challenges to integration that the authors had to resolve was the use of a common identifier when a same object is referenced in different repositories. Such problematic is not currently found in seismic data, since disparate repositories do not store information for the same objects.

Bio2RDF is another effort from the domain of bioinformatics that aims for the integration of data in the life sciences [31]. It is also based on RDF and provides a simple convention guideline for the creation of RDF data, to allow the integration of the multiple datasets. An online Website provides access to the integrated datasets, offers a data browser and provides access to related query services [32]. In comparison to our approach, Bio2RDF is again putting greater emphasis on the integration of distinct identifiers across datasets. There is also a large difference in scale, since Bio2RDF comprises more than 11 billion facts, while we are dealing with much smaller datasets. This leads to different technical requirements for the practical realisation.

Patton et al. [33] discuss SemantEco, an infrastructure to integrate distributed environmental and ecological data, in which different domains of data, called modules, can be dynamically added and removed. While modules are a flexible solution to implement different domain data, describing modules metadata adds an extra layer of complexity that is not currently needed in seismic hazard mitigation disciplines. However, it might be a useful design concept if seismic data has to be integrated with other disciplines in future. SemantEco utilises a family of ontologies to integrate data from different sources (mainly bird and fish observation, water quality criteria and the health effects of pollution on species) and extract new information to support decision systems. The authors highlight the capacity of ontologies to extend a data model easily, versus more traditional data management approaches such as the relational model. This is something that has been also experienced in Celestina, the system presented later on in this paper. Scalability of SemantEco has been considered, especially in terms of adding new data sources that can be queried in an ontological manner. Specifically, the authors mention D2RQ [34] as an interface to mediate between SemantEco and the recently added relational database. Finally, the discussion emphasises the relevance of having a distributed system instead of a centralised solution, in order to provide robust systems to manage large amounts of integrated data.

These examples have in common that they use distributed data sources, they are supported by the Web and use Semantic Web technologies for their integration efforts.

\subsection{Future developments for earthquake engineering data}

Experimental data originating from earthquake engineering projects is an ideal case for exploring and promoting the publication, sharing and integration of scientific data. Researchers have been sharing and reusing such experimental data in isolated situations for decades, but the great potential for scientific advances that benefit society, through safer and resilient infrastructure, is strong motivation to formalise such efforts. Simplistically, in terms of data, the first step for an institution is to have a repository, or a formalised way to store and manage data. The second step is to be able to feed the repository and create data (a task that has proved more difficult than expected when it comes to persuade users to organise and share data), potentially with the help 
of auxiliary tools to support the data input and their visualisation. The third step is to be able to share data with other institutions in a formalised, automated way. The fourth step is to be able to fully interoperate with any other institution and to construct global services for the community. In earthquake engineering, different institutions are at different stages, and there are still significant gaps in the community's capability. A concerted effort is needed to promote international collaborations in order to set the community moving in the same direction and to set institutions in the same stage.

Desirable features of experimental data and their social and technical infrastructure include the use of standards, the promotion of open data and policies to make experimental data available for human and machine consumption, the establishment of data provenance policies to provide credit to authors, the creation of infrastructures that allow laboratories and institution to keep control of the data they create, the establishment of close collaborations between IT experts and civil engineers, the creation of infrastructures that maximise the importance of the user experience $(\mathrm{UX})$, and mechanisms to produce trusted data.

\section{A PLATFORM FOR DATA INTEROPERABILITY}

Over the premises discussed before, we have worked on Celestina, a platform for the integration of hazard mitigation resources. This section discusses the subsystem called Celestina Data, which allows earthquake engineering institutions to exchange experimental data and consists of: (i) A data model to formalise the experimental knowledge gathered by research institutions, by using two of the main earthquake engineering data models as reference (the one in SERIES and the one in NEES). Its ultimate objective is pursuing the integration of experimental data in earthquake engineering globally. (ii) A distributed infrastructure, which deals with the systems and policies to implement the data model in the earthquake engineering institutions and to make practical use of the data integration. The infrastructure of Celestina Data is built from Celestina nodes, which are the distributed data sources deployed at the different earthquake engineering institutions.

Celestina Data is built using Semantic Web technologies. The Semantic Web is part of the Data Web initiative of the World Wide Web Consortium (W3C), ${ }^{7}$ which aims at enabling online data sharing and reuse through a variety of technology standards and best practices. Celestina Data leverages several technologies that are central to this effort: the graph-based data format $R D F$ (used to manage and exchange data), the ontology language $O W L$ (used to encode schema information to facilitate interoperability), and the query language SPARQL (used to retrieve information). We will briefly introduce the necessary concepts below. A more detailed first introduction is provided by Hitzler et al. [35].

The Semantic Web is a well established initiative, with strong industry support involving many organisations, and with an intensive, active research programme [36]. The associated technologies are particularly well-adapted to information integration in an open Web infrastructure, since they combine a number of basic principles and advanced features that are useful in this context. Important characteristics include the following:

7

https://www.w3.org/2013/data/ 
1. Global identifiers. Both the elements in a Semantic Web database and the vocabulary terms used to describe them are identified by URIs (Universal Resource Identifiers [37]). This popular standard facilitates exchange and recombination of data, since every URI identifies a unique object, irrespective of the context in which it is used. This is in contrast to identifiers in traditional databases, which only have a local meaning within a single database or application.

2. Schema flexibility. Semantic Web data does not require a fixed schema to be specified upfront, and data encoded in a variety of ways can co-exist within one application. This also makes it easy to extend or update the data encoding in the running system without necessarily breaking existing applications.

3. Declarative schema alignments. Ontologies are information models in which one can describe relationships between different ways of encoding similar data. This starts on the terminological level. For example, SERIES uses the term computation in the same way as NEES uses the term simulation. Ontologies empower users to specify such relationships without having to modify the underlying data encoding or software.

4. Handling incomplete data. A particular strength of ontology-based tools is that they can work with partial information. When integrating data from different sources, it is possible that one source expects details that the other does not provide. In such cases, rather than just omitting missing data entirely, ontologies can express the fact that some (unknown) data are known to exist.

5. Open standards. The above features might be realised in a number of data integration platforms. The advantage of Semantic Web technologies is that they rely on openly available standards that are supported by a variety of free and commercial software tools. This helps to build an open data ecosystem which ensures cross-platform interoperability and wide re-use (no technological lock-in).

An immediate benefit of following this approach is the availability of many mutually compatible software tools for a variety of tasks. (i) Storing and querying large amounts of RDF data is routinely handled by a range of RDF database management systems (colloquially called triplestores) such as Stardog [38], BlazeGraph [39] and Virtuoso [40]. (ii) OWL ontologies, which provide the organisational information models at the heart of our approach, can be created in tools such as Protégé [41]. (iii) Logical reasoning over such ontologies can then enable information integration, quality control and query answering, using OWL reasoners [42, 43, 44] or ontology-based query answering systems [45, 46]. Moreover, many RDF databases also have their own built-in lightweight OWL reasoning support, and Stardog in fact bundles a complete OWL reasoner to support even expressive ontologies.

\subsection{The Celestina Data data model}

The Celestina Data data model can be seen as a superset of the SERIES and NEES ${ }^{8}$ data models shown in Fig. 1, which are implemented in relational databases and have been used as a reference for data requirements. Both models store similar data structures but have quite different approaches in their data modelling. One of the main differences is that NEES considers specimens (the subject of the experiment) as attributes of experiments and simulations, whereas SERIES understands specimens as a hierarchy level itself over which experiments and

\footnotetext{
$8 \quad$ Note that although NEES is a finished project and its data model is likely to change in future projects, its data requirements still remain useful as a reference.
} 
computations are conducted. There is no right or wrong in this definition, and it is just a matter of how specimens are perceived in an experiment - whether many experiments are usually conducted over the same specimen or whether each experiment normally uses a different specimen. This hierarchy has less relevance in the data model of Celestina Data, since it is graphbased and specimens and experiments are linked in both directions.
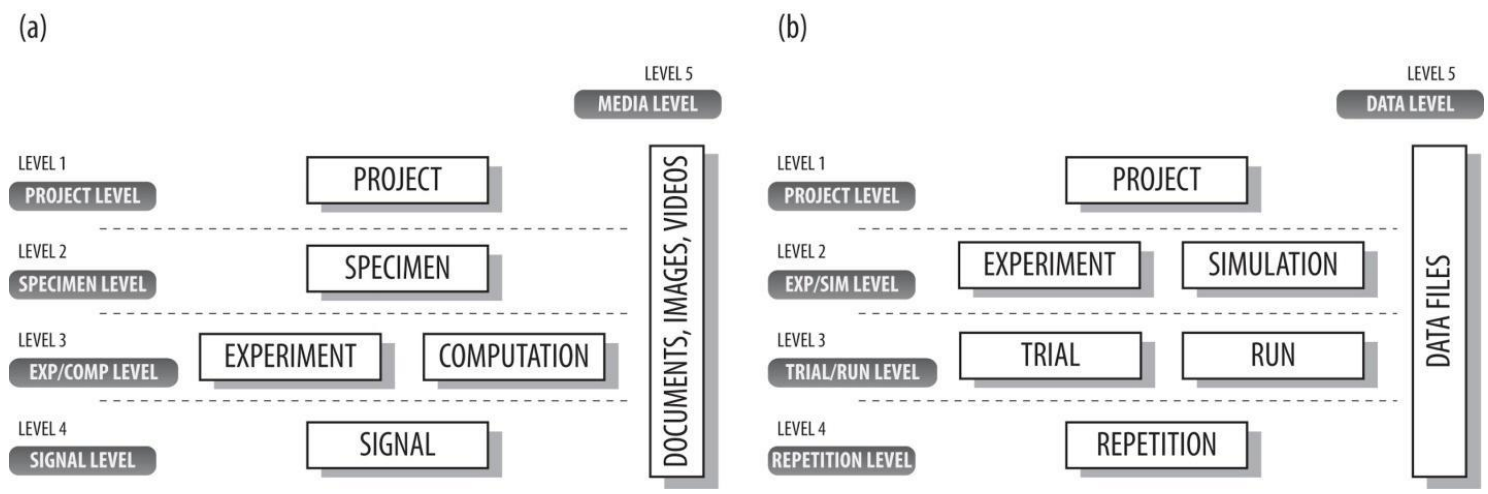

Fig. 1. (a) SERIES and (b) NEES data model hierarchies

The Celestina Data data model is implemented by means of an ontology. Generally speaking, this is an information model to capture a shared understanding of a domain of interest for a particular application [47, 48, 49]. The ontology of Celestina Data is specified using the OWL Web Ontology Language, an open standard of the World Wide Web Consortium that is used to encode ontologies in a machine-readable format [50]. An OWL ontology consists of a set of statements (called axioms) that define concepts and the specific relationships between them. The main elements of OWL are classes, properties and individuals. Classes are sets of resources with similar characteristics, such as the concepts of Earthquake Engineering Project, Specimen and Material. Individuals are members of classes (and individuals that belong to a class are called instances or objects of that class), for example a specific specimen tested within a project. Properties define binary relations, such as the property name that assigns a name to a project. The ontology defines relations such as the equivalence between similar concepts. So, for example, it is possible to use the terms "computation" and "simulation" obtaining equal results, since both terms are defined in the ontology as experimental activities of a numerical nature.

An important feature of OWL is its logical semantics, which establishes formal rules for the interpretation of ontologies in order to infer logical consequences and detect errors. This is called reasoning and tools providing such services are known as reasoners. Reasoning can lead to less maintenance effort and better quality control. For example, if our data state that a person is a test agent in an experiment, and this experiment is conducted in a project, a reasoner can infer that the person participates in the project. Ontologies can also be used to specify potential errors that should be detected automatically. For example, we might assert that a structure cannot be precast concrete and cast-in-place concrete at the same time, and a reasoner would be able to detect this issue if erroneous data are being entered.

The challenge in ontology construction is that it requires in-depth domain knowledge as well as significant technical expertise in the development of OWL ontologies. To address this, we performed the following steps for constructing the Celestina Data data model ontology: 
1. Expert consultations. Individual consultations with subject-matter experts from various subfields within the domain were used to acquire a first draft of the domain model and its representation in current databases. For this we consulted with selected experts from four different institutions (University of Oxford, Purdue University, the Joint Research Centre and Vienna Consulting Engineers).

2. Community enquires. In cases where the small group of experts cannot provide all information necessary to construct a model, it is necessary to involve the wider community. In our case, we conducted a survey to clarify the experimental terminology used in US and European projects, as explained below.

3. Ontology formalisation. The informal domain model that was acquired by the first two steps have then been formalised in OWL. Protégé has been used to create and edit the ontology.

4. Quality assurance. The constructed ontology was logically analysed using the HermiT reasoner, which is well integrated with Protégé. This helped to detect formalisation errors that led to undesired consequences, such as unexpected relationships between classes or, in extreme cases, inconsistency.

These steps were iterated as necessary. In particular, the attempt to formalise ontologies (Step 3) often reveals open issues that need to be addressed by returning to the experts (Step 1). Likewise, errors found in quality assurance (Step 4) must lead to changes in the formalisation (Step 3).

Obtaining useful feedback from the wider community (Step 2) can be difficult and this instrument should therefore be used with consideration. It is most effective when there is a clear question on which many participants can provide feedback. In our case, we found that the experts did not agree (or were unsure) regarding the meaning of certain experimental terms. We conducted a survey to clarify the situation. Participants were asked to group nine types of empiric investigation(experiment, simulation, test, computation, hybrid simulation, hybrid test, pseudo dynamic test, pseudo dynamic test with substructuring, pseudo dynamic test without substructuring)according to their modality (physical, numerical, a combination of both or unknown). The survey was answered by 27 earthquake engineers from 20 different institutions (12 EU, 8 USA). While some terms were clearly understood, the terminology about pseudo dynamic tests with/without substructuring was unclear to many participants. We have therefore decided to keep the term pseudo dynamic test, but exclude these other two terms from the initial data model, since there is no shared understanding of their meaning within and across the projects.

An important characteristic of our methodology is that it delegates the task of ontology construction to a small group of modelling experts who acquire the necessary domain knowledge by interacting with experts from the field. In our view, this is the most suitable approach for creating an initial prototype that shows the benefits of semantic technologies in a new field. When the use of these technologies becomes an integral part of the work in a project, it is important to transfer ownership of the ontological model to the subject-matter experts, e.g., by making ontology maintenance part of existing working groups that specify changes to the internal data models when needed.

The result of applying this methodology in our application area is the Celestina Data ontology, which we make available online. ${ }^{9}$ Based on the insights gathered during its 
construction, we have divided it into different sub-groups, in a similar fashion as the hierarchies of NEES and SERIES data models. This division, depicted in Fig. 2, can be used to create different independent ontologies, which can be maintained separately and gathered together in a single ontology as imported ontology modules. The project module describes all general information about earthquake engineering projects, which have objectives, start and end dates and outcomes. The project module subtly links to the specimen and the equipment and facilities modules, but its main link is with the experimental activity module. In this sense, the ontology is experimental-activity centred, and projects can be considered as high-level groups of experimental activities. The experimental activity module describes all physical and numerical experimental activities. Experimental activities are conducted under some loading parameters (input experimental information) and produce some results (output experimental information). This information is described in the experimental input/output module. Experimental activities are conducted over a specimen. Specimens are described in the specimen module, and the composition of the specimen is described in the materials module. Finally, experimental activities are supported by experimental equipment and systems. These are described in the facilities and devices module. There are two vertical modules that can complete information in the rest of the modules. The persons and organisations module describes participants for any task or activity. This can define the participation of persons and organisations in any module of the ontology, as for example, funding organisations for projects, principal investigators, test agents, people that constructed a specimen, etc. The media and data module describes documents, images, videos and other large data files that are used from the other modules. This division defines a high-level perspective of experimental data, which can probably be easily accommodated to experimental data of other hazard mitigation disciplines.

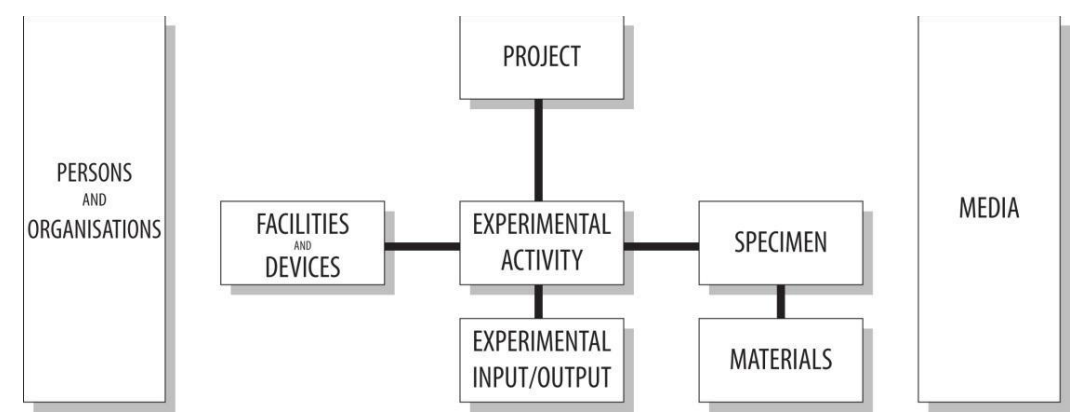

Fig. 2. Celestina Data ontology modules division

In total, the Celestina Data ontology consists of 1,140 axioms, 156 classes, 21 individuals, 67 object properties and 34 data properties. A detailed break-down into modules is given in Table 1. Note that some properties are shared between different modules and have been counted in more than one module, so the sum of the number of properties (and therefore, the sum of the number of axioms) does not match the numbers for the full ontology.

Taxonomies (hierarchies of concepts) used in the ontology, especially for materials and specimen component types, have been kept as simple as possible, since it has been experienced that development of an extremely complete taxonomy could lead to unnecessarily complicated systems that are rejected by their own users [51]. Therefore, a simpler yet useful data model is more beneficial than a complicated data model that is not used in practice. However, the Celestina Data ontology is by no means a complete and closed model. As any data model, it 
should be continuously developed, maintained and evolved to adapt it to current data needs in the earthquake engineering community.

\begin{tabular}{|c|c|c|c|c|c|c|}
\hline & Nr. axioms & Nr. classes & Nr. properties & Nr. object prop. & Nr. data prop. & Nr. individuals \\
\hline Celestina Data (full) & 1140 & 156 & 101 & 67 & 34 & 21 \\
\hline Project module & 129 & 2 & 16 & 7 & 9 & 3 \\
\hline Experimental activity module & 136 & 13 & 19 & 8 & 11 & 0 \\
\hline Experimental input/ouput module & 94 & 11 & 14 & 6 & 8 & 0 \\
\hline Specimen module & 230 & 58 & 9 & 5 & 4 & 0 \\
\hline Materials module & 140 & 22 & 10 & 5 & 5 & 0 \\
\hline Facilities and devices module & 247 & 31 & 29 & 14 & 15 & 0 \\
\hline Persons and organisations module & 51 & 4 & 9 & 5 & 4 & 0 \\
\hline Media and data module & 341 & 15 & 47 & 29 & 18 & 18 \\
\hline
\end{tabular}

Table 1. Statistical data about the Celestina Data ontology

\subsection{The Celestina Data infrastructure}

The Celestina Data ontology serves as the foundation for building applications to work with experimental data, but it needs an underlying technical infrastructure to organise the access to the different data sources. The general structure of Celestina Data is depicted in Fig. 3, which presents a high-level view of Celestina Data in four layers. To illustrate the extensibility of our approach, the figure also shows components related to wind engineering that have not been implemented in our prototype. It would be natural to add experimental data related to other hazards or to other types of experimental engineering data in general.

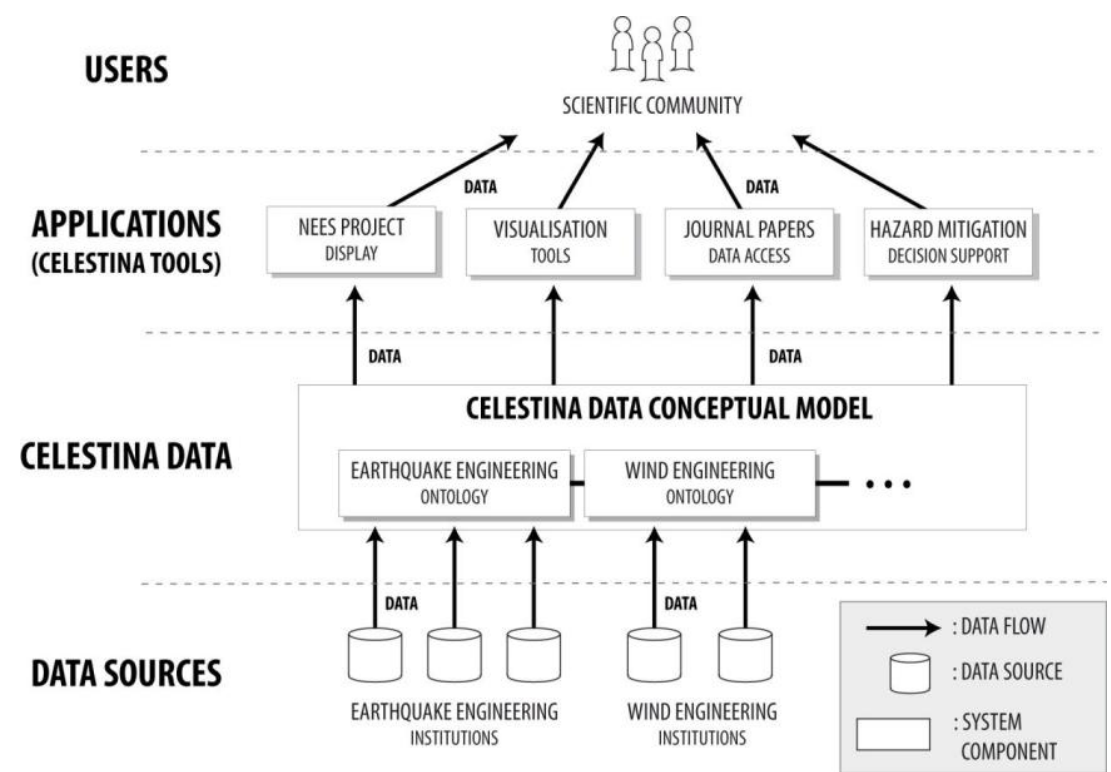

Fig. 3. Celestina Data four layer structure

The four layers in Fig. 3 show basic relationships between the main components and actors involved in Celestina Data. The bottom layer contains the data sources in their original formats, 
such as the relational databases in NEES and SERIES, or any other data source that should be integrated. The second layer is the heart of the Celestina Data infrastructure, which consumes data from the bottom layer, integrates it to obtain a unified view, and provides high-level access to the data to the application layer above. The work of this layer is centred around the Celestina Data model ontology described previously. The subsequent application layer, also called Celestina Tools, contains software tools that use the integrated data. These tools provide concrete benefits to users. Example applications shown in Fig. 3 include project-specific displays, visualisation tools, data repository services and decision support tools. Finally, the uppermost layer represents the users, primarily coming from the scientific community, who are the consumers of the data.

The core task of Celestina Data is data integration, which can be divided into two separate tasks. First, the content of the data sources must be represented in a unified form. Existing data sources are usually stored in legacy databases that may use a variety of formats and schemas. Second, the converted data must be conceptually integrated by aligning different views and terminologies of the data sources, so that users can get a coherent view of all available information. The Celestina Data model ontology is essential in both of these data integration phases: for the first phase, it defines the necessary vocabulary that is used to represent data in RDF (explained below); for the second phase, it specifies mapping rules that integrate different data.

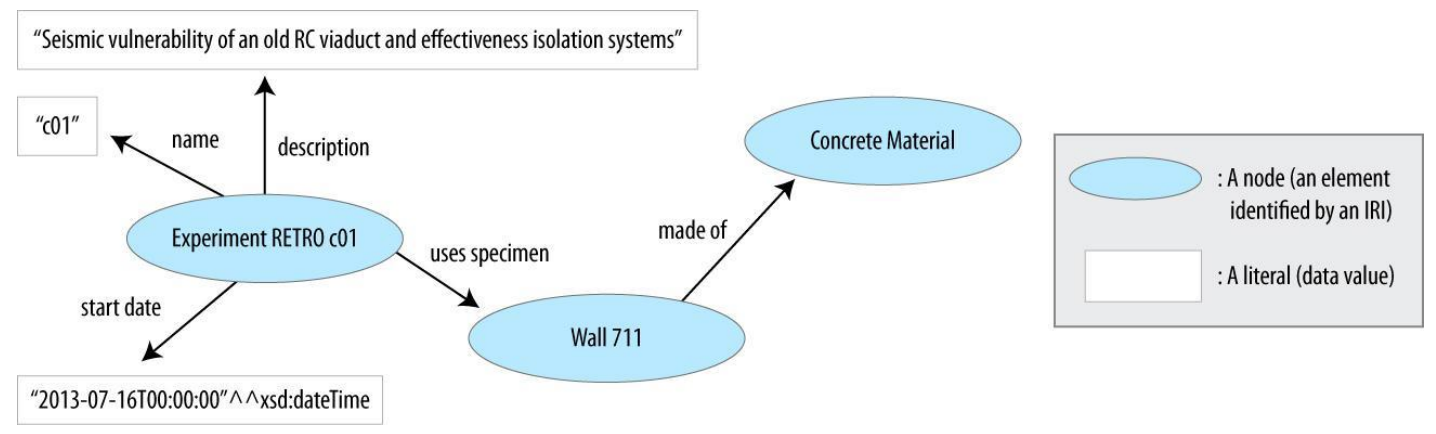

Fig. 4. An RDF graph

RDF (Resource Description Framework [52]) is the Semantic Web technology used to represent data in Celestina. It provides a graph-based data model. RDF graphs consist of a collection of nodes that are connected by labelled, directed edges, as illustrated in Fig. 4. Each edge expresses a relationship (specified by the label) between a source and a target node. The label of an edge is called property in RDF, and the edges may be viewed as assigning one or more property values (target nodes) to a source node. RDF nodes can have several forms and the standard supports a rich set of datatypes to express concrete data values (called literals in RDF). RDF is compatible with the OWL language that we use for the Celestina Data model ontology: both formalisms describe data by using properties in a similar fashion, and the membership of elements in an OWL class can likewise be expressed using a special property $r d f$ :type that connects an element to its class.

Small RDF graphs can be visualised as in Fig. 4, but real databases with large numbers of edges are encoded in more efficient, machine-readable formats. Typically this is done by storing the graph as a list of edges, where each edge can be encoded as a triple that consists of a subject, 
predicate and object, which denote the source, property, and target of an edge, respectively. This is why RDF databases are also called triplestores.

There are several methods for realising the two-step data-integration process that is performed in Celestina Data. Query-driven approaches consider the high-level user query and try to answer it by fetching information from the data sources, using their native access methods (e.g., relational queries). In this approach, the RDF representation of the data may remain virtual: it defines the interface to the ontological model, and thereby enables conceptual integration, but it is not necessary to store all data in this format. A popular implementation of this approach is ontology-based data access (OBDA [53, 54]), where declarative mapping rules are specified to define how relational data should be transformed into RDF. Given an ontology, OBDA systems can then automatically mediate between user queries and underlying data. This approach has been the subject of recent applied research projects such as OPTIQUE [55] and is supported by a significant number of tools $[34,45,56,57,58]$.

OBDA has many advantages, but it is also limited to a rather restricted subset of the OWL ontology language. Several features that are used in the Celestina Data model ontology are not supported. We have therefore decided to follow another route based on an extract-transformload (ETL) approach. We extract data from the original sources, transform this data to RDF, and load it into a dedicated RDF database. User queries are then performed over this database, and the ontology is taken into account by the database to obtain integrated results. This latter approach is called ontology-based query answering (OBQA [59]). We used the Stardog database for OBQA since it supports a wide range of OWL features, with a configurable trade-off between completeness and performance. The transformation of relational data to RDF can be achieved in many different ways; Sahoo et al. [60] give an overview. A standard language to help with the export of relational data into RDF is R2RML (RDB to RDF Mapping Language), as described by [61]. An alternative method is a direct mapping, as described by [62], which we have used in our work. Details are described in Section 4.3 below.

To realise this approach in Celestina Data, there exist three main roles:

a) Celestina Data nodes, which are the institutions that offer data services for the applications. Each of these nodes provides data in a conceptual data model of Celestina Data (an ontology). They host the data sources of Fig. 3. As a minimal requirement, nodes have to provide access to relevant parts of their data in RDF format that uses terms from the Celestina Data model ontology. This can be provided as a plain Web-accessible RDF file. Additional services, such as interactive query services, might be desirable but are optional. Internally, nodes can use very different hardware and software configurations. Celestina Data does not intend to replace existing (relational) data systems but to add a high-level, unified view to access heterogeneous data sources conceptually. For the nodes with existing databases, such as the ones at SERIES and NEES, we have generated RDF in an extract-and-transform approach as outlined above.

b) Celestina central site, which is a neutral, centralised entity in charge of serving the different ontology modules and storing the official directory of Celestina Data nodes. It hosts a Website that serves as the primary information point for the whole Celestina platform, and provides policies on how the data are licenced and how they should be cited and used. These policies, together with the capacity of the ontology to describe data provenance, work towards the support of authorship of data. The Celestina central site also contains the most updated versions of all ontological modules, such as the Celestina Data ontology discussed previously. It should be 
noted that Celestina Data is based on a versioned, centralised ontology, which has less overhead and is much easier to implement by multiple institutions than multiple distributed ontologies. However, this is mostly transparent to the user. In the cases of disparity of data, data that are only relevant for a single institution and concept disagreement, site-specific ontologies can be developed locally at each node. Finally, the central site maintains a list of official Celestina Data nodes and their contact details in a resources ontology. The decision of having a centralised directory of nodes, instead of other network structures such as a decentralised schema, is based on management requirements: (i) Data sources are expected to be continuously available and need to be monitored. (ii) If data have to be curated, a directory of data sources can help to identify nodes to which certain quality assurance policies should be applied. (iii) A centralised management is also more appropriate to verify global consistency in the data. Having a centralised directory presents a single point of failure, so Celestina Data nodes are encouraged to cache the served resources, especially the ontological modules and the list of nodes.

c) Applications, which are any external system that makes use of the services provided by a Celestina Data node, and constitute the gate to Celestina Data services for the end user. These systems can often be found inside the technical infrastructure of a Celestina Data node. Applications accessing data might need to connect to the Celestina central site to obtain the list of nodes that provide data services. Then, they can decide which of these nodes they need to access, connect each of the nodes directly, collect the data, process them and present them to the users of the application in a user-friendly way. The data updating process lies on the applications. Since Celestina Data is publicly accessible, applications are suggested to incorporate politeness, meaning that responsible access to Celestina Data nodes must be done by avoiding the generation of unlimited requests to the nodes. Note that this is regardless of the measurements that Celestina Data nodes have to implement to deal with impolite applications.

Since Celestina Data is a system for the inter-communication of computers, it has no direct human constituents or roles.

\subsection{Implementation}

Due to the architecture of the system, the implementation of an arbitrary data node is fairly simple. Although there exist more complex node implementations, as long as there is a Webaccessible RDF file with data in the Celestina Data ontology format, the node can be part of Celestina Data. The implementation of a Celestina Data node from the SERIES database implemented at the University of Oxford has been done. The software created is automatically extendable to any other SERIES node, and a similar infrastructure could be used at NEES or any other node with an existing relational database.

The first component implemented is the data export (extract and transform), which is responsible for exporting database data into an RDF file, by mapping SERIES data into the Celestina Data ontology format. The actual data export has been conducted by a direct mapping reusing the SERIES Virtual Database (SVDB) components that access the SERIES database. New code has been developed when SERIES private database tables had to be exported for Celestina Data; for example, device and sensor information is always private in the SVDB, but it is part of the public data in Celestina Data. No information explicitly marked as not public has been exported. The export of some SERIES data into the Celestina Data ontology has not been completely straightforward, and has been accomplished in a best-effort manner. For example, 
output experimental files are defined per experiment in the SERIES database, so it cannot be distinguished to which input information they belong to in an experiment with several input configurations. In this situation, each experimental input has been linked to all the experimental outputs within the experiment. Likewise, the relationship between input files and devices does not exist in the SERIES database. Also, classification of materials was done by guessing the material type according to a string search, and sensors were not sub-classified because SERIES data do not provide enough hints to attempt an accurate classification. These experiences do serve to inform future data model implementations.

To efficiently keep an updated version of the RDF file, the creation process of the file is triggered by external applications. To increase efficiency and to avoid race conditions (the possibility of incorrect results due to unlucky timing, as defined by [63]) a cache with mechanisms that guarantee mutual exclusion (a locking strategy that prevents two processes to access the same resource at the same time) has been implemented. The cache is created the first time the data are requested, and then it is regenerated every time the cache is considered expired according to some time threshold. By using a cache, the node does not have to access the SERIES database for every request, so requests can be served very efficiently and performance is dictated by the time it takes to download the RDF file through the network.

Large files such as documents, images and videos are downloaded from the Celestina Data node on request from the end user. In the ontology, the actual data is referenced by a download URI that normally points to a Web server on the node. SERIES implements some security mechanisms that have been adapted for the correct operation of Celestina (see [10] for a description of the download security mechanism implemented in SERIES). This implies to include Celestina as a trusted institution for the download of data.

The second significant implementation has been the Celestina central site. Because of the Celestina neutrality, it has been decided to host the Celestina central site in an external commercial hosting.

To provide information of all nodes serving Celestina Data and how to access their services, a simple resources ontology has been developed. Therefore, the whole system uses the same data technology. The central site also serves the Celestina Data ontology, and other ontologies (e.g. data models for other hazard mitigation disciplines) can be easily added by making them Web-accessible and linking them from the resources ontology.

\subsection{Validation}

The Celestina Data prototype has been validated by creating a Celestina Data application and testing it in a real environment between the University of Oxford (in UK), Purdue University (in Indiana, United States, where the NEES headquarters was located) and EUCENTRE (In Italy). The application consists of the presentation of data from Oxford and EUCENTRE in the NEES repository Website. This application provides NEES users with more data, with the advantage that users can interact with the new data by using the interface they are more accustomed to. A simplified schema of the environment for this application can be found in Fig. 5. The figure depicts four Celestina nodes: The Celestina central site, in a neutral location, and the Celestina Data nodes at NEES (Purdue University), Oxford and EUCENTRE. In this application, the nodes at Oxford and EUCENTRE provide an external access for their Celestina Data services, which are accessed by the NEES node. The latter does not need to provide any Celestina Data services for the validation so, for simplification purposes, no external access at NEES is shown in the figure. 


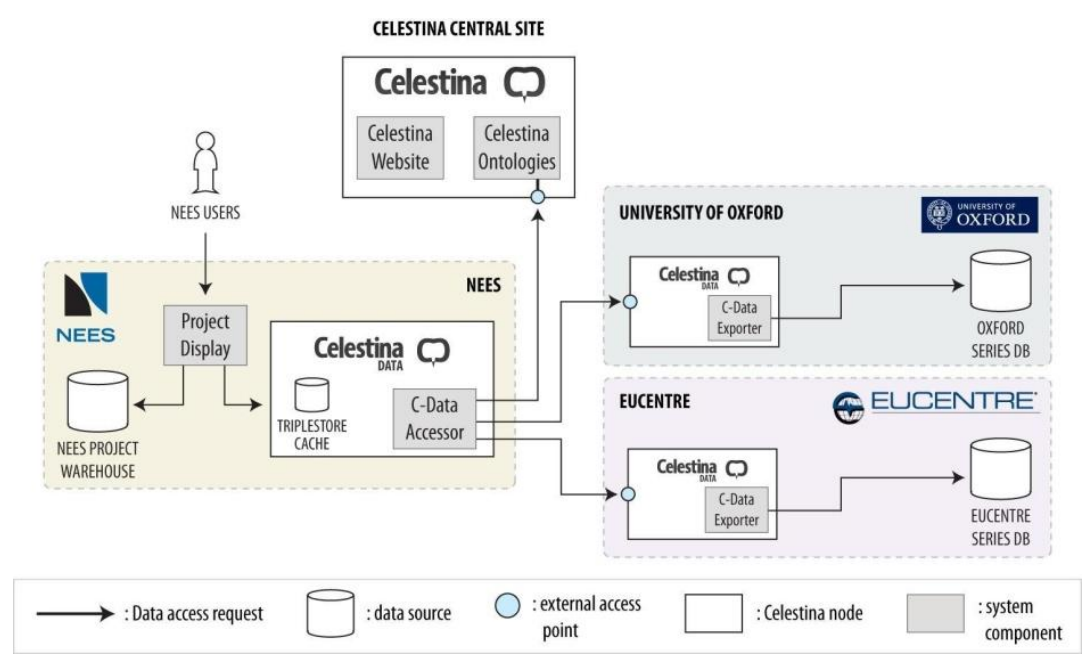

Fig. 5. Celestina Data validation environment between the universities of Oxford and Purdue and EUCENTRE

Nodes at Oxford and EUCENTRE use identical configurations with a component C-Data Exporter, which implements the data export previously discussed. The node at NEES uses a component C-Data Accessor, which is in charge of accessing the Celestina central site to query the ontology resources, cache it if necessary (to avoid the centralised single point of failure in case the central site is not responding in future), and access each of the nodes of the network. Note that the list of nodes can be dynamically updated at any time. As depicted in Fig. 5, the application works in this way: (i) In predefined periods of time, the C-Data Accessor contacts the Celestina central site and obtains the resources ontology. (ii) It extracts Celestina Data nodes' IRIs from the ontology and accesses each of them, including the Celestina central site to obtain the Celestina Data ontology (exceptions can be added about the nodes that are contacted). (iii) With all data collected, the application stores them in a Stardog triplestore that acts as a local cache. This is done to minimise request time when NEES users access Celestina data from the NEES Website, which is especially important when querying multiple geographically dispersed nodes on the network. (iv) When a NEES user accesses the public experimental data from the NEES Website, the NEES repository is accessed as usual, together with the local triplestore containing Celestina data, which is queried by using the SPARQL query language [64]. SPARQL is a standardised protocol and query language that is widely used to retrieve information from RDF databases. (v) Data from both sources, NEES and Celestina, are presented to NEES users in a unified manner. An example of result of the prototype can be found in Fig. 6, which depicts the NEES Website showing data from Oxford that has been accessed and integrated via Celestina. 


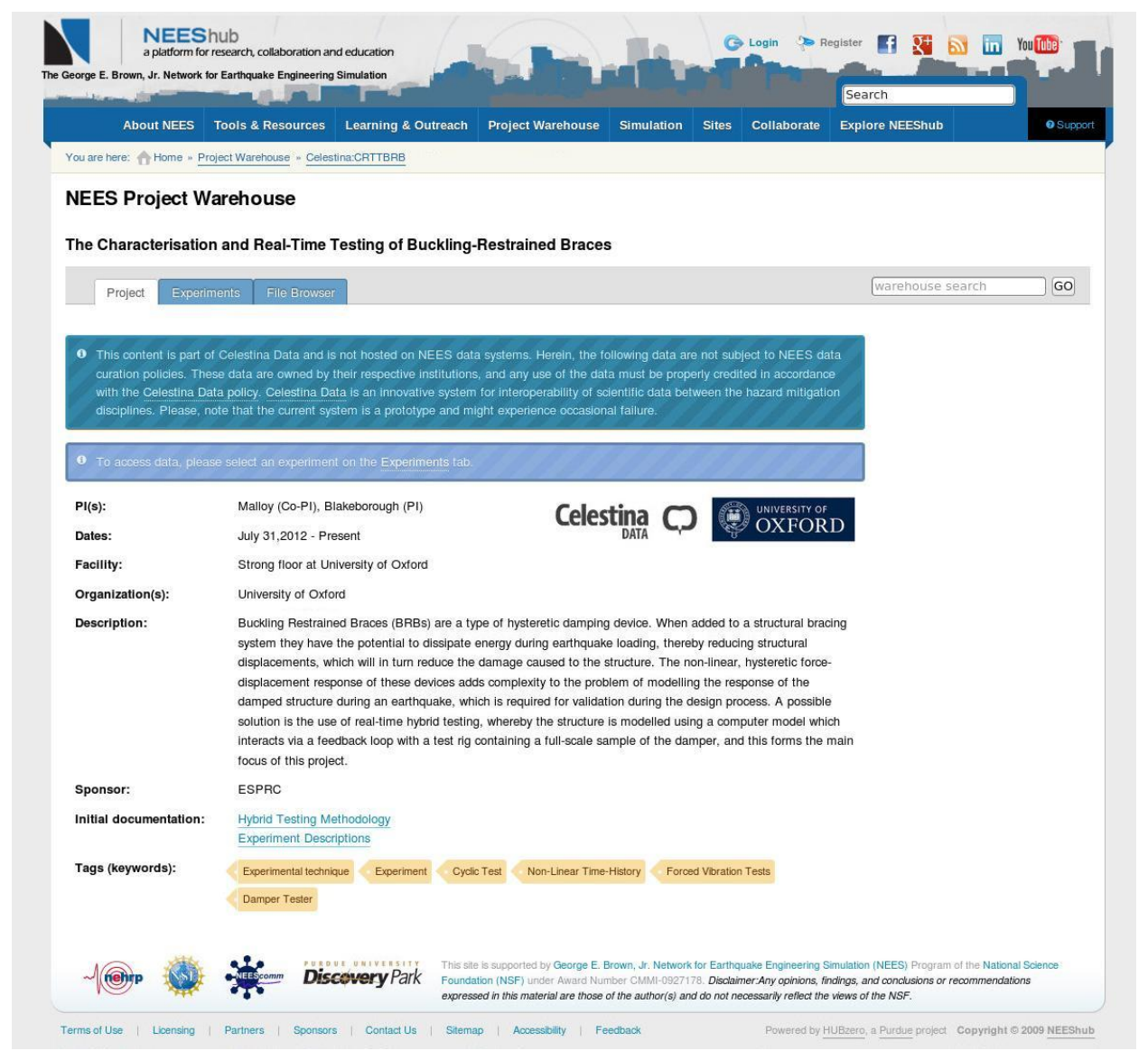

Fig. 6. Data from Oxford is shown in the NEES Cyberenvironment

\section{DISCUSSION}

The application discussed in the previous section has demonstrated the potential of a data integration effort between earthquake engineering institutions. The NEES Website has been automatically connected with more data from distributed sources, which benefits both sides: NEES obtains more data for their users without having to conduct the experiments or learn to use new data portals, whereas Oxford and EUCENTRE widen the reach of their research findings to other parts of the community without the need to develop Web interfaces or attract users. Moreover, once the infrastructure has been created, no effort is required to enable existing Celestina Data nodes to access data from new Celestina Data nodes. This means that if the components used at Oxford and EUCENTRE are installed in other SERIES institutions, NEES will automatically obtain the new data without any development or reconfiguration. As a result, the public subset of the 1,980 experimental activities from SERIES could join the 1,475 curated and public experimental activities of NEES (as of July 2014). As verified in other distributed environments, errors were sometimes very complex and tedious to trace. Some of the results are briefly discussed in the following sections.

\subsection{Complexity of node implementation}

The complexity of implementing a Celestina node primarily lies in the export process. Some parts of the SERIES data model were not directly translatable into the Celestina Data model represented by an ontology. The ontology is a more complete data model, and some of the 
information did not exist in SERIES data. However, this is not problematic since incomplete information is expected in RDF/OWL. Creating a bespoke exporter helped to refine the data presented in RDF, making more correct exporting decisions than those normally taken by automatic tools

\subsection{Performance}

Data served by both nodes was quite small, but significant enough to draw some conclusions. The node at Oxford served a total of 4,645 axioms in one project containing 21 experimental activities, whereas the node at EUCENTRE served a total of 5,900 axioms in three projects containing 9, 6 and 5 experimental activities. Best performance results were obtained when using a cache, with the Stardog triplestore loading data from and storing data on the disk (instead of the memory), taking a total time of 15.422 seconds to collect and process the data from both nodes. Loading triplestore times were acceptable for the system operation but not as a waiting time for a user. This confirms that using a triplestore as a cache is necessary. Although the response time was considered fast, it must be considered that a total of only four projects with 41 experimental activities were sent. If Celestina Data is expanded to other nodes around the world, the total response time could grow substantially. As an example of how these data can grow, project-level data from NEES have been exported to RDF. The time required to collect only project-level data of NEES public projects, consisting of 5,728 axioms, was 19 seconds. As a conclusion, it is clear that extensive use of caching techniques (on both client and server sides) is crucial to meet acceptable response times in Celestina Data, and this need will become more pressing as the number of Celestina Data nodes increases. This is in agreement with the findings of other data integration projects, such as Bio2RDF, which are also based on pre-loaded data exports rather than importing live data from original sources at runtime. A performance improvement that can be done in a Celestina external application is to run several threads to access different nodes in parallel, taking advantage of parallel processing at each node.

\subsection{Benefits of a Semantic Web data model}

In the modelling aspect, working with RDF/OWL showed little difference with respect to the creation of a relational data model. In both cases, data requirements were needed from experts of the domain, and several iterations were needed until the data model was considered accurate and stable. However, despite the fact that the relational model has been used successfully in all known earthquake engineering repositories, there are some issues that are addressed more efficiently by Semantic Web technologies, especially when it comes to achieving interoperability of disparate, geographically located data sources, for example:

a) Semantic Web technologies provide a common programming interface to retrieve knowledge from world-wide earthquake engineering laboratories. The information is published in a standardised way, supported by well known technologies, which makes data collaboration easier. b) Data integration is performed within the data model; no external programs are required. The technology is designed to facilitate data integration from different sources, which has simplified the infrastructure of Celestina Data and supported data interoperability that could not have occurred otherwise (for example, in the case that every other Celestina Data node had to implement SERIES Web Services).

c) Semantic Web technologies enable interoperability with a neutral, standard, technologyindependent framework to define data models. The result is that it is easier to import and export 
$\mathrm{RDF}$ data to/from different triplestores than to perform the equivalent operations with relational data between different relational database management systems. Despite the standardisation of the relational model, in practice, many relational database management systems still have many differences.

d) Because Semantic Web technologies are prepared to deal with incomplete data, an institution could generate information which can be completed by another institution. On the other hand, relational databases assume that data are complete (what is not in the database is considered false).

e) Data identifiers are universal in the Semantic Web, which is useful for example on data from distributed simulations. Part of the data can be stored in a node and be referenced from another node. This is difficult to accomplish in relational databases, because the identifiers have only a local meaning, and computers do not identify a similarity between the Experiment tables of two different databases.

f) Semantic Web data do not have a rigid, fixed structure. The information structure is very flexible, permitting changes and expansion. No fixed attributes exist as in a relational table, which benefits information that is difficult to store in fixed-length database rows. This also allows experts of a domain to create data structures without the need of a technology expert to modify the data schema.

g) Semantic Web technologies use reasoning to create new data from existing data. Also, data could be transmitted more compactly over the network and additional data can be inferred at the destination.

h) The Semantic Web is Web based, and is well integrated with other Web technologies. The Web has been demonstrated to be a robust platform from client to server sides.

i) Semantic Web concepts can have equivalent names and help with terminology agreements. Where a relational table will be called Experiment in the data model, a Semantic Web concept could use equivalent classes for Experiment, Test and Physical Experimental Activity, and users can utilise the term they are more accustomed with.

\section{FUTURE WORK}

Although the Celestina Data prototype shows great potential, many steps remain before hazard mitigation is established as a fully interoperable discipline. More international collaborations would have to be created with data sharing as an ingredient, and the interoperability effort has to be expanded to three areas: (i) Within other earthquake engineering institutions, such as other European SERIES institutions, the US institutions in NHERI, Korea, Japan, Taiwan, New Zealand, etc. (ii) To sibling hazard mitigation disciplines, such as seismology or coastal and wind engineering. As an example for the European context, OSAP ${ }^{10}$ collects information about buildings (materials, construction year, etc), NERA ${ }^{11}$ collects real-time seismic activity (accelerometers) and SERIES conduct experiments on a laboratory that can reveal the behaviour of certain materials under some seismic stimulus. By merging these three sources, risk assessment can be improved and decision support can be enhanced. (iii) To other industries, such as publishing. Using an interoperable data framework brings enriched content, context and

\footnotetext{
10 http://osap.faw.at/

11 http://www.seismicportal.eu/
} 
presentation of journal articles. This provides a better experience for readers and reviewers, who could directly view or investigate data in a standard format, facilitating greater understanding and validation of the research outcome.

More applications have to be developed under the Celestina platform. The prototype has shown integrated data from NEES and SERIES sources, but this is just one possible application. The potential of future applications based on Celestina Data is very large, and they have been grouped under a subsystem called Celestina Tools. Each and every Celestina tool can be reused automatically by any Celestina node, without the need of different institutions to each develop similar tools and duplicate effort. Note that such tools could be reused by nodes over their own repositories, without the need to share their data with a third party. Currently, the Joint Research Centre is working on an application to visualise and manage Celestina data.

The creation of the Celestina Data ontology is not a one-off development. As any other data model, the Celestina Data ontology must evolve with time, and has to be maintained, expanded and improved. To achieve this task, it is suggested that a Celestina Data consortium be created. This consortium should be comprised of a small group of both IT specialists and civil engineers from the main participant institutions of Celestina Data, with the mission of watching over general data requirements for the earthquake engineering community, and particularly over any required modifications of the Celestina Data ontology. One important consideration is that Celestina must be a neutral, community-driven platform, and, likewise, the consortium must be a neutral management body to represent the community. Celestina is by no means an infrastructure that is imposed by any institution, but a technical infrastructure to promote collaborations and joint efforts to address common, complex problems within the hazard mitigation disciplines. It is suggested that the consortium establishes common policies in terms of: implementation of Celestina nodes, curation, data licence and security. These should be recommendations rather than contractual obligations, and the consortium should provide support and guidance to Celestina nodes in these issues.

Achieving true interoperability in a scientific discipline is not an easy task, and requires effort from many participants in the field. World-wide data integration is technically challenging, although feasible. However, it is probably a more complex task to deal with the social challenges involved, because many disparate institutions are involved. Despite the patent benefits of interoperability, common interests and international agreements have to be met in an environment where funding bodies are not shared. Worldwide interoperability should be a short term feature of nearly every scientific discipline. Since technical challenges are no longer an impediment, it is a matter of having the willingness to join international coordinated efforts to achieve it.

\section{CONCLUSIONS}

This paper has discussed a vision for the future development of earthquake engineering experimental data, with a special focus on addressing the current lack of international interoperability, and keeping pace with the rapidly accelerating trend towards Open Data access. A prototype infrastructure for the interoperability of seismic experimental data has been created and validated between the University of Oxford, NEES (Purdue University) and EUCENTRE. Technologically, the Semantic Web has been used to support the infrastructure, and an ontology has been created for the integration of data. The next steps include the implementation of this effort to link other earthquake engineering institutions around the world, the expansion to other industries and sibling disciplines, and the creation of Celestina-based tools that can provide 
tangible benefits to the end user. These capabilities will enable a new generation of earthquake and multi-hazard engineers to have ready access to important experimental data sets for accelerating research on resilience.

\section{ACKNOWLEDGMENT}

The work presented here was supported in part by the U.S. National Science Foundation (under award number CMMI-0927178) and the European Union's Seventh Framework Programme (under grant agreement no. 227887). The authors would like to acknowledge the support of Purdue University staff during the development of the Celestina Data prototype, especially to Prof. Julio Ramirez, Standa Pejša, Gemez Marshall, Brian Rohler, Limin Dong, Richard White, Barbara Fossum and the members of the Core feedback group of NEEScomm. We also would like to acknowledge the support of EUCENTRE members Francesco Lunghi and Davide Silvestri during the validation of the system.

\section{REFERENCES:}

[1] Spencer, B.F. and Leon, R.T. (eds.) (2006) World Forum on Collaborative Research in Earthquake Engineering: An Invitational Workshop, San Francisco, California, March 16-18. 2006.

[2] NEES Consortium, Inc.,(2007) Information Technology within the George E. Brown, Jr. Network for Earthquake Engineering Simulation: A Vision for an Integrated Community, NEES 07-01, April 2007.

[3] Hacker T. J., Eigenmann R., Bagchi S., Irfanoglu A., Pujol S., Catlin A., Rathje E. (2011) The NEEShub Cyberinfrastructure for Earthquake Engineering. Computing in Science \& Engineering, 13(4):67-78.

[4] Pejša S., Dyke S. J., Hacker T. (2014) Building Infrastructure for Preservation and Publication of Earthquake Engineering Research Data. The International Journal of Data Curation, 9(2):83-97.

[5] Pejša S. (2012) NEEScomm requirements for curation and archiving of research data. https://nees.org/resources/4759 [February 2016].

[6] Pejša S., Hacker T. (2013) Curation of Earthquake Engineering Research Data. https://nees.org/resources/5751 [February 2016].

[7] Pejša S., Weisman D. (2011) Data Sharing and Archiving Policies. https://nees.org/resources/2811 [February 2016].

[8] Howlett G. A., Catlin A. C., Nasi K., Howell R. (2009) inDEED. http://nees.org/resources/indeed. [February 2016].

[9] Dong L., Mathew I., Rodgers G. P. (2014) PEN 2.4. https://nees.org/resources/pen. [February 2016].

[10] Lamata Martínez I., Ioannidis I., Pegon P., Williams M., Blakeborough A. (2013) The Process and Future of Data Integration within the European Earthquake Engineering Laboratories. J. Comput. Civ. Eng., 10.1061/(ASCE)CP.1943-5487.0000308.

[11] Research Councils UK. http://www.rcuk.ac.uk [August 2016].

[12] Wellcome. Policy on data management and sharing. http://www.wellcome.ac.uk/About-us/Policy/Policy-andposition-statements/WTX035043.htm [August 2016].

[13] National Science Foundation. Public access to results of NSF-funded research. http://www.nsf.gov/news/special_reports/public_access/index.jsp [August 2016].

[14] Open Energy Data. http://www.energy.gov/data/open-energy-data [August 2016].

[15] NIH. Public Access policy. http://publicaccess.nih.gov [August 2016].

[16] HEFCE. Policy for open access in the post-2014 Research Excellence Framework http://www.hefce.ac.uk/pubs/year/2014/201407/ [August 2016].

[17] NSF (2015) Open Government Plan 3.5 June 2015.http://www.nsf.gov/pubs/2015/nsf15094/nsf15094.pdf [May 2016].

[18] BioMed Central https://www.biomedcentral.com [August 2016].

[19] PLOS. https://www.plos.org [August 2016].

[20] PMC. http://www.ncbi.nlm.nih.gov/pmc [August 2016].

[21] Europe PMC. https://europepmc.org [August 2016].

[22] OpenAIRE. https://www.openaire.eu [August 2016].

[23] Data.gov. The home of the U.S. Government's open data. https://www.data.gov [August 2016]. 
[24] European Governments, open data. https://data.gov.uk, https://www.data.gouv.fr, etc. [August 2016].

[25] Asian Governments, open data. http://www.data.go.jp, https://data.gov.in, etc. [August 2016].

[26] NEES Licensing content - Intellectual property considerations. https://nees.org/legal/licensing [August 2016].

[27] European Plate Observing System. (2016) Legal framework. https://www.epos-ip.org/node/167/pdf [October 2016]

[28] ORFEUS (2016) Guidelines for becoming an ORFEUS EIDA primary or secondary node.

http://www.orfeus-eu.org/data/eida/eida_guidelines.html [October 2016]

[29] Hacker T. J., Dyke S. J. (2015) Cyberinfrastructure to Empower Scientific Research. Proceedings of the Whither Turbulence and Big Data, Springer, Corsica, France, April 20-24, 2015.

[30] Deus H. F., Prud'hommeaux E., Miller M., Zhao J., Malone J., Adamusiak T., McCusker J., Das S., Rocca Serra P., Fox R., Marshall M. S. (2012) Translating standards into practice - One Semantic Web API for Gene Expression. Journal of Biomedical Informatics, 45(4):782 - 794

[31] Callahan A., Cruz-Toledo J., Dumontier M. (2013) Ontology-Based Querying with Bio2RDF's Linked Open Data. J. Biomedical Semantics 4(S-1): S1 (2013)

[32] Bio2RDF. Linked Data for the Life Sciences. http://bio2rdf.org/ [August 2016].

[33] Patton E.W., Seyed P., Wang P., Fu L., Dein J., Bristol S., McGuinness D.L. (2013) SemantEco: A semantically powered modular architecture for integrating distributed environmental and ecological data. Future Generation Computer Systems, Elsevier.

[34] D2RQ (2013) The D2RQ Platform: Accessing Relational Databases as Virtual RDF Graphs. http://d2rq.org [February 2016].

[35] Hitzler P., Krötzsch M., Rudolph S. (2009) Foundations of Semantic Web Technologies. Chapman \& Hall/CRC. ISBN: 9781420090505.

[36] Horrocks I. (2011) Tool Support for Ontology Engineering. In Dieter Fensel, editor, Foundations for the Web of Information and Services, pp. 103-112. Springer.

[37] Berners-Lee T., Fielding R., Masinter L. (2005) Uniform Resource Identifier (URI): Generic Syntax. RFC 3986. http://tools.ietf.org/html/rfc3986 [February 2016].

[38] Stardog. http://stardog.com/ [August 2016].

[39] Blazegraph. https://www.blazegraph.com/ [August 2016].

[40] Virtuoso. http://virtuoso.openlinksw.com/ [August 2016].

[41] Protégé. http://protege.stanford.edu/ [August 2016].

[42] Glimm B., Horrocks I., Motik B., Stoilos G., Wang Z. (2014) HermiT: An OWL 2 Reasoner. J. Autom. Reasoning 53(3), 245-269.

[43] Steigmiller A., Liebig T., Glimm B. (2014) Konclude: System description. J. Web Sem. 27, 78-85.

[44] Kazakov Y., Krötzsch M., Simancik F. (2014) The Incredible ELK - From Polynomial Procedures to Efficient Reasoning with EL Ontologies. J. Autom. Reasoning 53(1), 1-61.

[45] Calvanese D., Cogrel B., Komla-Ebri S., Kontchakov S., Lanti D., Rezk M., Rodríguez Muro M., and Xiao G. (2016) Ontop: Answering SPARQL Queries over Relational Databases. Semantic Web Journal.

[46] Zhou Y., Nenov Y., Cuenca Grau B., Horrocks I. (2014) Pay-as-you-go OWL query answering using a triple store. In Proceedings of the Twenty-Eighth AAAI Conference on Artificial Intelligence, 2014, 1142-1148.

[47] Horrocks I. (2013) What are ontologies good for?. Evolution of Semantic Systems, pp. 175-188. Springer.

[48] Guarino N., Oberle D., Staab S. (2009) What is an ontology. Handbook on Ontologies, Staab S., Studer R. (eds.), Springer-Verlag, 2nd ed., 2009, pp. 1-17.

[49] Studer R., Benjamins R., Fensel D. (1998) Knowledge engineering: Principles and methods. Data \& Knowledge Engineering, 25(1-2):161-198.

[50] Hitzler P., Krötzsch M., Parsia B., Patel-Schneider P. F., Rudolph S. (2012) OWL 2 Web Ontology Language Primer (Second Edition). W3C Recommendation. http://www.w3.org/TR/owl2-primer/ [February 2016].

[51] Bardet J. P., Liu F., Mokkaram N. (2004) A metadata model for the George E. Brown Jr. Network for Earthquake Engineering Simulation. Report, Civil Engineering Department, University of Southern California.

[52] Schreiber G., Raimond Y. (2014) Resource Description Framework (RDF) 1.1 Primer. W3C Recommendation. http://www.w3.org/TR/rdf11-primer/ [February 2016].

[53] Poggi A., Lembo D., Calvanese D., De Giacomo G., Lenzerini M., Rosati R. (2008) Linking data to ontologies. Journal on Data Semantics X, 133-173.

[54] Kontchakov R., Lutz C., Toman D., Wolter F., Zakharyaschev M. (2011) The combined approach to ontologybased data access. Proc. 22nd Int. Joint Conf. on Artificial Intelligence (IJCAI'11). pp. 2656-2661. AAAI Press/IJCAI. 
[55] Calvanese D., Giese M., Haase P., Horrocks I., Hubauer T., Ioannidis Y. E., Jiménez-Ruiz E., Kharlamov E., Kllapi H., Klüwer J. W., Koubarakis M., Lamparter S., Möller R., Neuenstadt C., Nordtveit T., Özçep Ö. L., Rodríguez Muro M., Roshchin M., Savo D. F., Schmidt M., Soylu A., Waaler A., Zheleznyakov D. (2013) Optique: OBDA Solution for Big Data. ESWC (Satellite Events), pp. 293-295.

[56] Pérez Urbina H., Motik B., Horrocks I. (2009) A comparison of query rewriting techniques for DL-lite. In Proc. of DL 2009, volume 477 of CEUR, ceur-ws.org.

[57] Calvanese D., De Giacomo G., Lembo D., Lenzerini M., Poggi A., Rodríguez Muro M., Rosati R., Ruzzi M., Savo D. F. (2011) The Mastro System for Ontology-based Data Access. Semantic Web journal, 2 (1), 2011. IOS Press.

[58] Erling O., Mikhailov I. (2013) Mapping Relational Data to RDF in Virtuoso. http://www.openlinksw.com/dataspace/doc/dav/wiki/Main/VOSSQLRDF [February 2016].

[59] Krötzsch M., Rudolph S. (2016) Is Your Database System a Semantic Web Reasoner?

KI, 30(2):169-176, Springer 2016.

[60] Sahoo S. S., Halb W., Hellmann S., Idehen K., Thibodeau T., Auer S., Sequeda J., Ezzat A. (2009) A Survey of Current Approaches for Mapping of Relational Databases to RDF. W3C Report. http://www.w3.org/2005/Incubator/rdb2rdf/RDB2RDF_SurveyReport.pdf [February 2016].

[61] Das S., Sundara S., Cyganiak R. (2012) R2RML: RDB to RDF Mapping Language. W3C Recommendation. http://www.w3.org/TR/r2rml/ [February 2016].

[62] Arenas M., Bertails A., Prud'hommeaux E., Sequeda J. (2012) A Direct Mapping of Relational Data to RDF. W3C Recommendation. http://www.w3.org/TR/rdb-direct-mapping/ [February 2016].

[63] Göetz B., Peierls T., Bloch J., Bowbeer J., Holmes D., Lea D. (2006) Java Concurrency in Practice. AddisonWesley Professional. ISBN: 978-0321349606.

[64] SPARQL (2013) SPARQL 1.1 overview. The W3C SPARQL Working Group. W3C Recommendation. http://www.w3.org/TR/sparql11-overview/ [February 2016] 